\title{
Shallow marine EM surveys
}

\author{
I.Ingerov \\ Advanced Geophysical Operations and \\ Services Inc. (AGCOS)
}

\begin{abstract}
Seabed EM surveys in shallow water environments have a number of specific particularities. Specifically, there is difficulty with hermetical sealing of EM instruments and the necessity of overcoming electromagnetic noise caused by underwater currents and sea surface disturbances. Another problem is the inability to employ large ocean going vessels in many areas, while the use of small vessels increases the demands on the size and weight of the equipment. There are also certain positive aspects in this, pertaining to the ability to use reliable acoustic communication between the marine EM instrument positioned on the seabed and the sea surface, as well as the use of beacons for identifying the location of the bottom apparatus. In this paper, discussed is a shallow marine EM data acquisition equipment complex that allows efficient use of standard ground EM instruments for carrying out investigations at a sea depth interval of $0-200 \mathrm{~m}$. This complex consists of $2 x-4 x-5$-channel marine EM systems.
\end{abstract}

Key words-Shallow marine, EM, seabedsurveys

\section{INTRODUCTION}

Over the past two decades, technologies and equipment have been created to conduct surveys using magnetotellurics (MT) and controlled sources EM in deep basins ${ }^{[1]}$. We have accumulated a wealth of positive experience in locating hydrocarbon deposits in various parts of the world, as well as mapping deep structures, including salt domes, faults, etc. However, these surveys have revealed a number of problems with ensuring profitability of marine EM surveys. Currently, vessel rental costs several thousand dollars a day, thus requiring concurrent deployment of several dozen seabed receivers in order to produce feasible soundings. Such surveys require significant capital investment ${ }^{[2]}$ Consequently, these surveys can only be profitable when exploring large areas. Another characteristic of deep-water equipment is its considerable weight and a availability of only 2- and 4-channel EM recording systems. It is difficult and ineffective to deploy large seagoing vessels in shallow waters. In such circumstances, it is much more effective to use small nimble vessels. Such an approach puts stricter demands on the requirements for dimensions and weight of marine equipment. As has been established in the first decade of the current century, the use of a vertical magnetic component of EM field can provide significant additional information ${ }^{[3]}$. This fact makes it desirable to include 5- or 3-channel receivers equipped with vertical magnetic sensors in the marine system complex. For shallow depth marine equipment, it is important to also consider two important factors affecting the quality of field recordings: sea movement and underwater currents ${ }^{[4]}$.

\section{II.METHOD}

Chinese geophysicists in the Gulf of Bohai were the first to experiment in shallow waters with Phoenix equipment, at the beginning of the current century ${ }^{[5]}$. Work was carried out from a fishing boat (Fig. 1, A) by stretching electrical lines using lifeboats. Despite a positive geological result, this methodology could not withstand serious criticism from the point of view of work safety, accuracy, metrology, productivity, performance and cost (Fig. 1, B).
A)

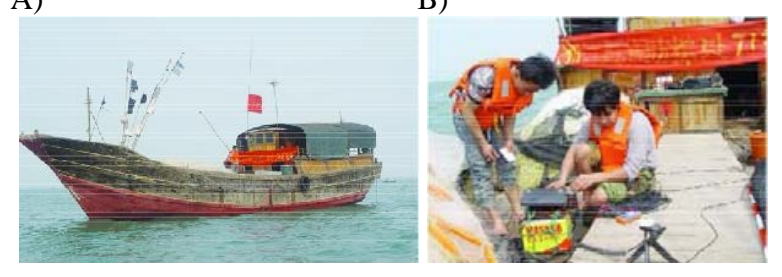

Fig. 1 A) The Chinese fishing trawler that served as the ZPX's "mother ship"; survey equipment was deployed from small motor boat; B) The ZPX crew prepares MT survey equipment on the trawler's deck.

In the construction of underwater equipment, there are two approaches. The first of these is the creation of a complete system that includes special EM field receiver. The second approach involves the use of well-known, wellproven receiver and magnetic components EM field sensors, and only requires the creation of hermetic enclosures, navigational systems, communication systems, and systems for deployment and resurfacing. This article chooses the second approach. The equipment designed by the author of this article has another particularity: two gradations of the equipment, with specific mechanical characteristics. That is, equipment for transition zones (depth of $0-50 \mathrm{~m}$ ) and equipment for the shelf (depth of $10-200 \mathrm{~m}$ ). In waters with significant depth fluctuations, it is possible to use the two types of equipment concurrently.

\section{III.SEABED EM SYSTEMS}

This is the more complex type of marine equipment as on the one hand, surveys with their use are carried out in environmentally sensitive basins and it is desirable to avoid leaving any environment footprints on the seabed. On the other hand, these systems have to work in conditions of heightened electromagnetic interference associated with the sea currents and waves, as well as interference from coastal power plants and electric signals from passing vessels. Hence the increased demands on the height and aerodynamics of the system enclosure (minimum resistance enclosure), and the inadmissibility of concrete - or other ballast (used as detachable anchors in marine systems). 
Stringent environmental requirements also eliminate the use of lead and its salts in the construction of porous nonpolarizing electrodes. The 2-channel shallow depth electromagnetic marine system SMMT was specifically designed, tested, and put into production specifically for surveys in shallow marine and freshwater basins with the depth interval of 0 to $50 \mathrm{~m}$ (Fig. 2).

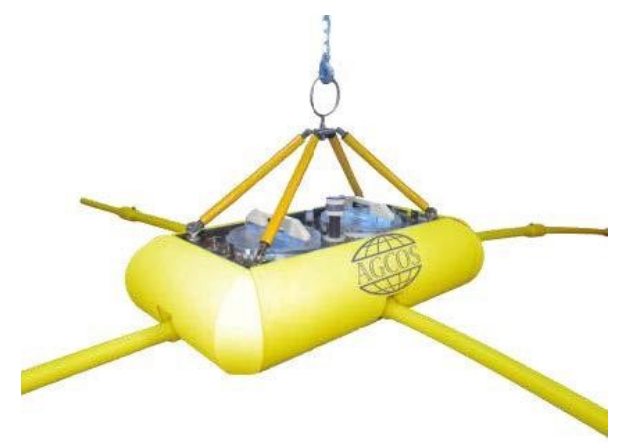

Fig. 2 Marine EM receivers for transition zone exploration; 2- channel SMMT.

It is important to note that this system is equally suitable for works on land areas subject to periodic flooding (tidal territory, estuaries, water surges, melt water pathways). As noted abo function EM field recorder and its battery source. The SMMT system has a very low height, provides low aerodynamic resistance, allowing two field crew members to move it effortlessly in its assembled form. The system was tested and showed good functionality with multifunction receivers MTU-2E, MTU-5A Gepard-4A Gepard-8A. The system can thus be used with conventional land EM field receivers produced by various geophysical companies.

The system has a sturdy frame, which mounts the aerodynamic enclosure and two hermetic cylinders containing the recording device, communication and alarm systems, navigational system, and a battery source. Four telescopic poles capped with non-polarizing electrodes are easily attached and detached using custom connectors and locks. The recesses built into the system contain the environmentally friendly recovery subsystem components that are conFig.d and controlled through on-deck user interface software for submersion or ascent of the SMMT system. In its submerged state, the system has an acoustic communication device, as well as an acoustic beacon designed to work for at least a month in case the acoustic communication device fails for some unexpected reason. In a surfaced state, the system will initiate GPS and visual beacons, as well as radio communication. The small size and weight of the system, as well as its detachable telescopic poles, allow for its use from small vessels (pontoon, catamaran, yacht) and tug up to 10 systems. The requirements for the vessel's crane system are also very reasonable: capacity 0.5 tones, swing radius $2 \mathrm{~m}$ overboard).

The system is deployed as follows: after fresh batteries and a removable media card with a recorded data acquisition schedule of operations are installed, the cylinders containing the equipment are hermetically sealed. The vessel reaches its measurement site location, the recording device is synchronized with GPS, and the communication and alerts systems are activated. With the help of a crane, the system is moved overboard, the electric line dipoles are attached, the system is lowered to the water surface, the recovery system is reset and the system gently sinks to the seabed (Fig. 3). At the appointed time (specified in the loaded schedule of operations), the system begins registering the EM field in sync (via GPS) with other submerged systems, as well as with a ground base station. System recovery, relocation to another survey point and consequent redeployment is then carried out.

\section{IV.SEABED EM SYSTEMS FOR COASTAL SHELF}

Seabed shelf systems are designed for works at the depth interval of 10 to $200 \mathrm{~m}$. 2- and 5-channel systems are shown in Fig. 4, A and Fig. 4, B respectively. The main structural element of each system is a frame made of durable nonmagnetic material. The main working components of the system are mounted onto the bottom part of the plastic frame. Two detachable high pressure spheres are attached to the bottom of the system. The recording device (2-, 4- or 8channel) is installed in one of these, along with the navigational, communication, and alarm systems. The second sphere houses independent batteries for the EM field receiver and for each system. The spheres are connected to each other by high pressure marine cables. Four telescopic poles capped with multi- stage, low noise, non-polarizing electrodes are also attached to the plate. The poles are connected to the sphere housing the receiver with high pressure marine cables. A detachable concrete anchor is mounted to the bottom of the plate, which gives the system negative buoyancy. The marine system is equipped with acoustic communication and alert systems, as well as a radio communication system that activates once the system resurfaces. The inside of the spheres also contains sensors that help the controlling instrument register depth, system azimuth, degree of inclination, temperature, humidity inside the sphere, and other parameters. System buoys are mounted to the top part of the frame and balance the system underwater. The buoy containing a strong cable and which ascends on command is also housed here. Adjustable legs are mounted to the frame and serve to install the system on the seabed and protect it from being suctioned to the muddy ground.

The 5-channel system houses three orthogonal magnetic sensors in custom high pressure enclosures. The sensors are connected to the sphere containing the EM receiver with deep sea high pressure cables. When requested, the 5channel system can be built to contain a device to automatically level the magnetic sensors. The system has several options of recovery. Via an acoustic signal received from the surface, the buoy containing the cable ascends to the surface. The cable automatically coils as the buoy ascends. A vessel approaches the surfaced buoy, hooks the cable onto the crane winch, and lifts the system via the cable to the surface of the sea and further via the crane to the deck of the vessel. If, for some reason, the surfacing buoy malfunctions, an acoustic signal is issued to release the concrete block. As the system has positive buoyancy without the concrete anchor, it then ascends to the surface at a moderate rate. Limited dimensions and weight of the system 
(160 kg 2-channel and $290 \mathrm{~kg}$ 5-channel) allow it to be used on relatively small vessels with a crane able to handle 2 tones and swing its arm $5 \mathrm{~m}$ overboard. The deployment of the system at Caspian Sea is shown Fig. 5 (First Marine MT Survey in Russia, 2009).

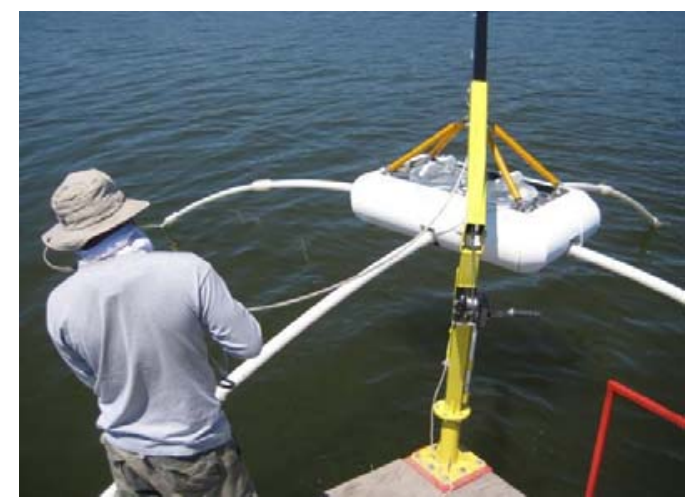

Fig. 3 Deployment of SMMT system during marine EM survey from the pontoon boat.

A)

B)
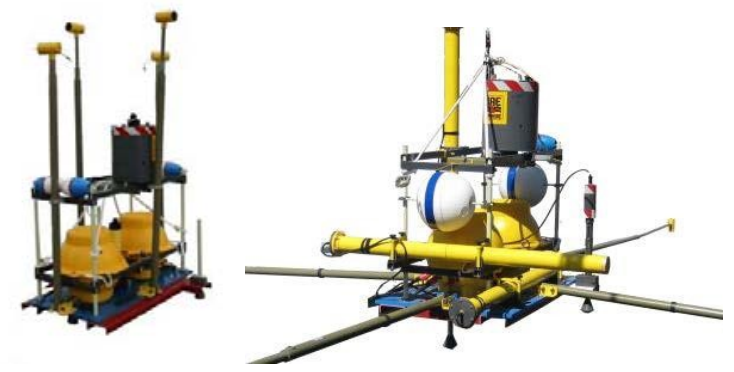

Fig. 4 Marine EM receivers for coastal shelf exploration; A) 2- channel 2AUSS-07A; B) 5-channel 5AUSS-07A.

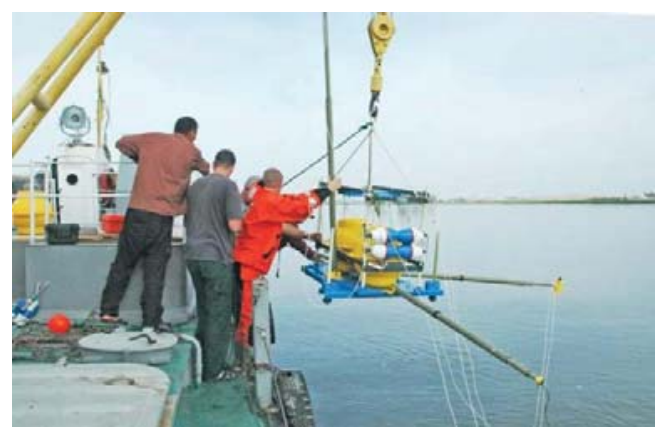

Fig. 5 Nord West Ltd. (Moscow, Russia) personnel deploy a shallowwater Marine MT system in the Caspian Sea.

\section{V.DEPLOYMENT AND REDEPLOYMENT OF MARINE EM RECEIVERS}

The crew required to service the marine systems consists of only three experts (two operators and one for data processing and analysis) and the weight of the system is limited (2ch- $160 \mathrm{~kg}, 5 \mathrm{ch}-290 \mathrm{~kg})$. As a result, the size of the required vessel can be quite small.

Main requirements for the vessels:

- Local safety regulations;

- Corresponding marine regulations and permits to be as far from the shore as the marine EM surveys require;

- Safe storage for at least 10 systems;

- Open deck space for at least one system operation (transfer from storage to working mode);

- Crane with at least 2000kg lifting capacity and an arm with an extension length of at least $5 \mathrm{~m}$ from the vessel.

Deployment of the system:

- Switch the system to a working position on the deck of a vessel;

- Insert a fresh battery pack and a removable media card with a loaded start-up table for the receiver;

- Turn on the EM receiver and the communication module; acquire GPS coordinates and a synchronization lock;

- Seal all pressure vessels;

- Using the vessel's crane, deploy the system in the water. Recovery of the system:

- Sail to the location where the system was deployed according to GPS coordinates;

- Establish acoustic communication with the system and acquire status parameters;

- Send "Release" command to disengage the pop-up buoy and wait for it to reach the surface;

- Fish out the cable attached to the pop-up buoy and attach it to the winch;

- Pull the system out of the water using the winch;

- Put the system on the deck and retract the telescoping booms;

- Open pressure vessels, extract the data, change the battery pack and the removable media card;

- Move the system to a safe storage space;

- Copy the acquired data to a PC for further processing.

If there is a problem with the pop-up buoy's response, an acoustic command to release the concrete anchor can be sent and system will surface by itself.

\section{VI.CONCLUSIONS}

Shallow depth seabed marine systems for recording EM fields at the seabed have been created and tested in the field. The limited size and weight of these systems, as well as their original design, allow them to be used by a small field team (3-5 persons, not including the vessel crew).

The systems described above allow for the use of small vessels, which significantly reduces the cost of the work and increased the maneuverability in shallow water.

- Deep water seabed marine EM systems are not economically viable for the exploration of coastal shelf and transition zones;

- Deep water marine EM receivers are not equipped with a vertical magnetic component that can provide important geological information;

- Specialized seabed marine EM systems, 2AUSS-07A and 5AUSS-07A (2 and 5 channel respectively), can be 
deployed/retrieved from relatively small vessels;

- Specifically designed for the exploration of transition zones, the SMMT system has a very low profile and an aerodynamic shape, resistant to surface waves and underwater currents. It is equipped with marine lownoise non-polarized pots and can be deployed from small vessels or catamarans;

- Marine EM surveys carried out from relatively small vessels with shallow seabed marine EM receivers can be profitable;

- The shallow marine EM systems can also be successfully applied to geological engineering and pipeline and underwater cables route investigations.

\section{REFERENCE}

[1] Chave, A.D., et al., 1991, Electrical Exploration Methods for the Seafloor, In: Nabighian, M. (Ed). Investigations in Geophysics, No. 3
- Electromagnetic Methods in Applied Geophysics, Vol.2, Application/Parts A and B, SEG publication.

[2] Farelly, B., et al., 2004, Remote characterization of hydrocarbon filled reservoirs at the Troll Field by Sea Bed Logging. EAGE Fall Research Workshop, Rhodes, Greece. First Marine MT Survey in Russia, 2009, The Phoenix (newsletter of Phoenix Geophysics Ltd.), Issue \#48.

[3] Fox, L., and Ingerov, O., 2007, Natural source EM for offshore hydrocarbon detection offers potential cost savings. First Break, Vol.25, Nov.2007.

[4] Marine MT in China with Phoenix equipment, 2004, The Phoenix (newsletter of Phoenix Geophysics Ltd.), Issue\#34.

[5] Vozoff, K., 1991, The magnetotelluric method. In: Nabighian, M. (Ed.) Investigations in Geophysics, No.3 - Electromagnetic Methods in Applied Geophysics, Vol.2, Aplication/Parts A and B, SEG publication. 